\title{
The quality of severe mental disorder diagnoses in a national health registry as compared to research diagnoses based on structured interview
}

Ragnar Nesvåg ${ }^{1 *}$ (D) Erik G. Jönsson 2,3, Inger Johanne Bakken ${ }^{1}$, Gun Peggy Knudsen ${ }^{1}$, Thomas D. Bjella², Ted Reichborn-Kjennerud ${ }^{1,4}$, Ingrid Melle ${ }^{2,4}$ and Ole A. Andreassen ${ }^{2,4}$

\begin{abstract}
Background: Utilization of diagnostic information from national patient registries rests on the quality of the registered diagnoses. We aimed to investigate the agreement and consistency of diagnoses of psychotic and bipolar disorders in the Norwegian Patient Registry (NPR) compared to structured interview-based diagnoses given as part of a clinical research project.

Methods: Diagnostic data from NPR were obtained for the period 01.01.2008-31.12.2013 for all patients who had been included in the Thematically Organized Psychosis (TOP) study between 18.10.2002 and 01.09.2014 with a Diagnostic and Statistical Manual of Mental Disorders, 4th edition (DSM-IV) diagnosis of schizophrenia $(n=537)$, delusional disorder $(n=48)$, schizoaffective disorder $(n=118)$ or bipolar disorder $(n=408)$. Diagnostic agreement between the primary DSM-IV diagnosis in TOP and the International Classification of Diseases, 10th revision (ICD-10) diagnoses in NPR was evaluated using Cohen's unweighted nominal kappa (K). Diagnostic consistency was calculated as the proportion of all registered severe mental disorder diagnoses in NPR that were equivalent to the primary diagnosis given in the TOP study.

Results: The proportion of patients registered with the equivalent ICD-10 diagnosis as the primary DSM-IV diagnosis given in TOP was $84.2 \%$ for the schizophrenia group, 68.8\% for the delusional disorder group, 76.3\% for the schizoaffective disorder group, and $78.4 \%$ for the bipolar disorder group. Diagnostic agreement was good for schizophrenia $(k=0.74)$ and bipolar disorder $(k=0.72)$, fair for schizoaffective disorder $(k=0.63)$, and poor for delusional disorder $(\mathrm{K}=0.39$ ). Among patients with DSM-IV schizophrenia, 4.7\% were diagnosed with ICD-10 bipolar disorder, and among patients with DSM-IV bipolar disorder, 2.5\% were diagnosed with ICD-10 schizophrenia. Diagnostic consistency was $84.9 \%$ for schizophrenia, 59.1\% for delusional disorder, $65.9 \%$ for schizoaffective disorder, and $91.0 \%$ for bipolar disorder.
\end{abstract}

Conclusions: When compared to research-based diagnoses, clinical diagnoses of schizophrenia and bipolar disorder in the NPR are accurate and consistent, with minimal diagnostic overlap between the two disorders.

Keywords: Psychosis, Schizophrenia, Bipolar disorder, Registry, ICD-10, DSM-IV

\footnotetext{
* Correspondence: ragnar.nesvag@fhi.no

${ }^{1}$ Norwegian Institute of Public Health, P.O. Box 4044Nydalen, N-0403 Oslo,

Norway

Full list of author information is available at the end of the article
} International License (http://creativecommons.org/licenses/by/4.0/), which permits unrestricted use, distribution, and reproduction in any medium, provided you give appropriate credit to the original author(s) and the source, provide a link to the Creative Commons license, and indicate if changes were made. The Creative Commons Public Domain Dedication waiver (http://creativecommons.org/publicdomain/zero/1.0/) applies to the data made available in this article, unless otherwise stated. 


\section{Background}

Data from national health registries represent a unique source for research into health service quality and disease epidemiology. Population-based health care registry data are particularly important for rare and severe diseases like psychotic disorders due to complete national coverage. When using such data, information about diagnostic accuracy and consistency is crucial. A review of studies evaluating diagnostic information in the Swedish National Inpatient Register concluded that the validity was high for most, but not all types of somatic and psychiatric diagnoses [1]. Systematic assessments of diagnostic accuracy in health registries in Denmark [2], Sweden [3, 4], and Finland [5-7] have generally shown good agreement between register diagnoses of schizophrenia spectrum disorders and research diagnoses based on case notes and/or structured diagnostic interviews.

In industrialized countries, the great majority of people with severe mental disorders will be in contact with public health care facilities at some point during their lifetime. Data from Israel indicate that $93 \%$ of patients diagnosed with schizophrenia in an epidemiological survey were also registered with schizophrenia in a national psychiatric registry [8].

The Norwegian Patient Registry (NPR) includes subjectspecific administrative and diagnostic information from 2008 and onwards from all governmentally funded specialist health treatment facilities, i.e. public hospitals, private clinics funded by the government, and private specialists who receive reimbursement for their treatment of patients. This includes the majority of all psychiatric treatment in Norway, as there are no psychiatric hospitals without government support, and only few fully private psychiatrists. In NPR the diagnoses are registered as International Classification of Diseases, 10th revision (ICD-10 [9]) codes as reported by the treating clinicians. In psychiatric care settings, diagnoses are typically determined by the physician or psychologist, either alone or after a discussion with colleagues and staff members. For psychiatric disorders, data from the NPR have previously been used to investigate prevalence of neurodevelopmental disorders among children [10], co-morbid substance use disorders in severe mental disorders [11], and prevalence of selfpoisoning with medications among adolescents [12]. The quality of mental disorder diagnoses in the NPR is at present unknown.

The aim of the present study was to investigate the quality of severe mental disorder diagnoses (schizophrenia, delusional disorder, schizoaffective disorder, and bipolar disorder) in the NPR by comparing diagnoses based on structured diagnostic interview and review of case notes as part of a clinical research project with diagnoses given by the treating clinicians and reported to the NPR. We specifically aimed to investigate diagnostic agreement and consistency. Our hypothesis was that a schizophrenia diagnosis would show better agreement than schizoaffective and bipolar disorder diagnoses.

\section{Methods}

\section{Data sources}

The study sample consisted of 1111 patients who were recruited to the Thematically Organized Psychosis (TOP) study between 18.10.2002 and 01.09.2014 and had consented to registry linkage. The TOP study was initiated at the University of Oslo in 2002 and is an ongoing multicenter, multidisciplinary investigation of clinical, genetic, neuroimaging, pharmacological and neurocognitive features of schizophrenia and bipolar disorders. Details regarding recruitment of patients and clinical procedures have been reported previously [13]. Briefly, patients with psychotic disorders were consecutively recruited from the major hospitals in the Oslo region and clinically assessed by trained research physicians, psychiatrists, and psychologists. All patients were diagnosed according to the Diagnostic and Statistical Manual of Mental Disorders, 4th edition (DSM-IV [14]) following a semi-structured interview [15] and review of case notes. The diagnostic assessment in the TOP study has very good reliability, and the overall agreement for the DSM-IV diagnostic categories tested is $82 \%$ and the overall Kappa 0.77 (95\% CI, 0.60-0.94) [13].

For the present study only the main diagnosis at the patients' first participation in the TOP study was used for comparison with registry data. The patients were grouped into four diagnostic categories, based on their primary DSM-IV diagnosis at inclusion to the TOP study: schizophrenia (including schizophreniform disorder) $(n=537)$, delusional disorder $(n=48)$, schizoaffective disorder $(n=118)$, and bipolar disorder (including bipolar disorder, type I, bipolar disorder, type II, and bipolar disorder, not otherwise specified) $(n=408)$.

The NPR is a national health registry covering all sectors of the governmentally funded specialized health care services in Norway, including somatic, psychiatric and substance use treatment facilities. All hospitals, clinics and private practitioners who receive governmental reimbursement are obliged to report activity and diagnostic data to the NPR. In Norway, there are publicly funded mental health care facilities for in- and outpatient treatment in all regions of the country. There is a maximum annual fee for outpatient treatment (in 2016: 2185 NOK, appr. $230 €$ ) while inpatient treatment is free of charge.

The unique 11-digit personal identification number has been reported for each patient to the NPR from 2008 onwards. This allows for linkage with other data sources as well as keeping track of individual disease trajectories across treatment facilities in Norway. 
For the present study, data from NPR for the period 01.01.2008-31.12.2013 were obtained for all patients within the four diagnostic categories defined above who had been recruited to TOP and consented to registry linkage. Data on hospital stays and outpatient consultations were retrieved from the NPR. All available NPR data were used irrespective of when the patient was included in the TOP study. We had information on the level of care (inpatient/outpatient), type of care (somatic hospital, mental health care facility, substance use treatment facility or psychiatrists/psychologists with governmental reimbursement), and assigned ICD-10 diagnoses. All ICD-10 codes for mental disorders (F00-F99) were included. Specifically, the study looked into four major severe mental disorder categories: schizophrenia (F20), persistent delusional disorder (F22), schizoaffective disorder (F25), and bipolar disorder (F30-F31). Additionally, to calculate diagnostic agreement and consistency, for the four main diagnostic categories, a broader group of «severe mental disorders» was formed which included schizophrenia-like psychotic disorder (F20-F29), bipolar disorder (F30-F31), and depressive disorder with psychotic features (F32.3, F33.3). To determine the number of specialist health care contacts, which included a psychiatric disorder diagnosis, a «mental disorder» category was formed, which included all F-codes in the ICD-10 (F00-F99).

\section{Statistical Analysis}

Differences in demographic and clinical data between the four diagnostic groups were analyzed using KruskallWallis equality-of-populations rank test with Dunn's post-hoc pairwise comparison. The number of registrations in the NPR with each of the selected ICD-10 categories was calculated for each of the four DSM-IV diagnostic groups.

Diagnostic agreement was calculated as the proportion of patients who were either correctly classified as having the diagnosis in question (schizophrenia, delusional disorder, schizoaffective disorder, or bipolar disorder), or correctly classified as not having the diagnosis in question. The precision of diagnoses in NPR compared to TOP was further evaluated using Cohen's unweighted nominal kappa ( $\mathrm{K})$, a metric that estimates overall agreement while taking into account the possibility of the agreement occurring by chance [16]. To evaluate the predictive properties of severe mental disorder diagnoses in NPR, sensitivity (i.e. the probability that an ill person will receive the correct diagnosis), specificity (i.e. the probability that a person without the illness will not receive the diagnosis), positive predictive value (PPV, i.e. the probability that a person diagnosed as ill is truly ill) and negative predictive value (NPV, i.e. the probability that a person not registered with the disorder truly did not have the disorder) were calculated. In the analyses of agreement, kappa, sensitivity, specificity, PPV, and NPV, the total group of 1111 patients were considered to be the target population, patients within one of the four DSM-IV diagnostic categories under scrutiny were considered to be truly ill, while patients with the remaining three diagnostic categories were considered to be "not ill". The occurrence of the equivalent ICD-10 diagnostic category at least once in the NPR was considered to be a positive test, while absence of registrations in NPR with the equivalent ICD-10 diagnostic category was considered to be a negative test.

Diagnostic consistency was calculated as the percentage of all registered contacts in NPR with a severe mental disorder diagnosis (i.e. ICD-10 codes F20-F29, F30F31, F32.3, or F33.3) that included the primary DSM-IV diagnosis assigned when recruited to the TOP-study.

To investigate if diagnostic agreement or consistency differed between sexes, we performed supplementary analyses of men and women separately. Since patients were usually recruited to the TOP study while in treatment in specialized health care, the diagnostic data from the TOP study and the NPR are not completely independent. The degree of dependency is presumably higher for patients recruited during the period NPR-data were available (2008-2013) than for patients recruited prior to this period. To test if time of recruitment affected the estimates of diagnostic agreement and consistency, we performed supplementary analyses after splitting the sample into two groups: patients recruited to TOP before NPR-data were available, i.e. before 01.01.2008, and patients recruited when subject-specific NPR-data were available, i.e. after 01.01.2008. All statistical analyses were performed in STATA 14 (StataCorp. 2015. Stata Statistical Software: Release 14. College Station, TX: StataCorp LP.)

\section{Results \\ Demographic and clinical data}

Demographic and clinical data for the 1111 patients are presented in Table 1 . There were significantly more men in the schizophrenia and delusional disorder groups compared to the schizoaffective and bipolar disorder groups. Mean age at inclusion to the TOP study ranged between 30.3 years for the schizophrenia group to 35.4 years for the delusional disorder group. Patients with schizophrenia or schizoaffective disorder had significantly more registrations with any or severe mental disorders, more hospitalizations, outpatient visits and bed-days compared to patients with delusional disorder or bipolar disorder.

\section{Diagnostic agreement}

The proportion of patients registered in NPR with the equivalent ICD-10 diagnosis as the primary DSM-IV 
Table 1 Demographic and clinical data for 1111 patients recruited to the Thematically Organized Psychosis (TOP) Study

\begin{tabular}{|c|c|c|c|c|c|c|c|c|c|}
\hline \multirow{2}{*}{$\begin{array}{l}\text { TOP-diagnosis (DSM-IV) } \\
\% \text { men }\end{array}$} & \multicolumn{2}{|c|}{ SCZ $(n=537)$} & \multicolumn{2}{|c|}{$\operatorname{DEL}(n=48)$} & \multicolumn{2}{|c|}{ SCA $(n=118)$} & \multicolumn{2}{|c|}{$\mathrm{BIP}(n=408)$} & \multirow{2}{*}{$\frac{\text { test }(p \text {-value })^{\mathrm{a}}}{.0001^{\mathrm{b}}}$} \\
\hline & 63.5 & & 64.6 & & 35.6 & & 40.0 & & \\
\hline \multirow[t]{2}{*}{ Age (mean, SD) } & 30.3 & 9.4 & 35.4 & 9.8 & 32.3 & 11.2 & 34.5 & 12.1 & $.0001^{\mathrm{c}}$ \\
\hline & Median & IQR & Median & $\mathrm{IQR}$ & Median & $\mathrm{IQR}$ & Median & $I Q R$ & \\
\hline Months of NPR data pre TOP & 2 & $0-21$ & 10 & $0-40$ & 2 & $0-21$ & 0 & $0-17$ & .0935 \\
\hline Months of NPR post TOP & 69 & $49-39$ & 61 & $31-74$ & 66 & $37-99$ & 71 & $54-96$ & $.0365^{d}$ \\
\hline $\begin{array}{l}\text { Any mental disorder } \\
\text { (ICD-10 F00-99) }\end{array}$ & 52 & $21-106$ & 27 & $10-79$ & 46 & $16-90$ & 26 & $5-61$ & $.0001^{\mathrm{e}}$ \\
\hline $\begin{array}{l}\text { Severe mental disorder } \\
\text { (ICD-10 F20-F31, F32.3, F33.3) }\end{array}$ & 45 & $13-97$ & 16 & $4-45$ & 37 & $10-76$ & 14 & $1-45$ & $.0001^{f}$ \\
\hline Hospitalizations & 3 & $1-7$ & 1 & $0-3$ & 4 & $1-9$ & 2 & $0-5$ & $.0001^{9}$ \\
\hline Outpatient visits & 72 & 34-137 & 48 & $28-90$ & 74 & $32-115$ & 43 & $16-84$ & $.0001^{h}$ \\
\hline Bed-days & 87 & $2-330$ & 11 & 0-91 & 61 & $4-257$ & 10 & $0-79$ & $.0001^{i}$ \\
\hline
\end{tabular}

Abbreviations: DSM-IV Diagnostic and Statistical Manual of Mental Disorders, 4th edition, SCZ schizophrenia, DEL delusional disorder, SCA schizoaffective disorder, $B I P$ bipolar disorder (type I, type II, and bipolar disorder not otherwise specified), SD standard deviation, IQR interquartile range, NPR Norwegian Patient Registry, ICD-10 International Classification of Diseases 10th revision

${ }^{a}$ Kruskall-Wallis equality-of-populations rank test with Dunn's post-hoc pairwise comparison

${ }^{\mathrm{b}} X^{2}=50.8$ (SCZ > SCA, $p<0.0001$; SCZ $>$ BIP,$p<0.0001$; DEL $>$ SCA,$p=0.0004 ; \mathrm{DEL}>\mathrm{BIP}, p=0.0005$ )

${ }^{c} X^{2}=31.9$ (DEL > SCZ, $p=0.0004 ; \mathrm{DEL}>\mathrm{SCA}, p=0.02 ; \mathrm{BIP}>\mathrm{SCZ}, p<0.0001 ; \mathrm{BIP}>\mathrm{SCA}, p=0.03$ )

${ }^{d} X^{2}=8.5(\mathrm{SCZ}>\mathrm{DEL}, p=0.009 ; \mathrm{SCA}>\mathrm{DEL}, p=0.01 ; \mathrm{BIP}>\mathrm{DEL}, p=0.002)$

e $X^{2}=66.4$ (SCZ > DEL, $p=0.002 ; \mathrm{SCZ}>\mathrm{BIP}, p<0.0001$; SCA $>$ DEL, $p=0.0411 ; \mathrm{SCA}>\mathrm{DEL}, p=0.0001$ )

${ }^{f} X^{2}=85.2$ (SCZ > DEL, $p=0.0003$; SCZ $>$ BIP, $p<0.0001$; SCA $>$ DEL, $p=0.01$; SCA $>$ BIP, $\left.p<0.0001\right)$

${ }^{9} X^{2}=35.3$ (SCZ > DEL, $p=0.0001 ; \mathrm{SCZ}>\mathrm{BIP}, p<0.0001$; SCA $>$ DEL, $p=0.0001$; SCA $>\mathrm{BIP}, p<0.0001$ )

${ }^{\mathrm{h}} X^{2}=64.9$ (SCZ > DEL, $p=0.02$; SCZ $>\mathrm{BIP}, p<0.0001$; SCA $>\mathrm{DEL}, p=0.04$; SCA $\left.>\mathrm{BIP}, p<0.0001\right)$

${ }^{\mathrm{i}} X^{2}=71.6$ (SCZ > DEL, $p=0.0001$; SCZ $>$ BIP,$p<0.0001$; SCA $>$ DEL, $p=0.001$; SCA $>$ BIP, $p<0.0001$ )

diagnosis given in TOP was $84.2 \%$ for the schizophrenia group, $68.8 \%$ for the delusional disorder group, $76.3 \%$ for the schizoaffective disorder group, and $78.4 \%$ for the bipolar disorder group (Table 2). In the schizophrenia group, $7.1 \%$ had received an ICD-10 diagnosis of schizoaffective disorder, and $4.7 \%$ had been diagnosed with bipolar disorder in the NPR. Conversely, in the bipolar disorder group, $2.5 \%$ had ever received an ICD-10 schizophrenia diagnosis, and $2.7 \%$ a persistent delusional disorder diagnosis in NPR (Table 2). Kappa reliability measures were higher for schizophrenia (0.74) and bipolar disorder (0.72) than for schizoaffective (0.63) and delusional disorder (0.39) (Table 3). Diagnostic specificity was high (0.90-0.94) for all diagnostic categories. PPV was high for schizophrenia and bipolar disorder $(0.88$ and 0.86 , respectively), moderate for schizoaffective disorder (0.61), and low for delusional disorder (0.31). NPV was high (0.86-0.97) for all diagnostic categories.

\section{Diagnostic agreement across gender and time of recruitment}

For schizophrenia and bipolar disorder, the diagnostic agreement was similar in men and women, while for delusional and schizoaffective disorder the sensitivity was higher among women than men (Table 3). Kappa was higher for men than women in all diagnostic categories except for bipolar disorder, where women had higher kappa than men. Kappa was higher for schizophrenia patients who were recruited prior to 2008 compared to schizophrenia patients recruited in 2008 or later, while

Table 2 Concordance ${ }^{a}$ between ICD-10 diagnoses in the Norwegian Patient Registry (NPR) and DSM-IV diagnosis in the Thematically Organized Psychosis (TOP) study

\begin{tabular}{|c|c|c|c|c|c|c|c|c|c|}
\hline \multicolumn{2}{|l|}{ TOP group } & \multicolumn{2}{|c|}{$\mathrm{SCZ}(n=537)$} & \multicolumn{2}{|c|}{ DEL $(n=48)$} & \multicolumn{2}{|c|}{$\operatorname{SCA}(n=118)$} & \multicolumn{2}{|c|}{$\mathrm{BIP}(n=408)$} \\
\hline NPR diagnoses & ICD-10-codes & $n$ & $\%$ & $n$ & $\%$ & $n$ & $\%$ & $n$ & $\%$ \\
\hline Schizophrenia & F20 & 452 & 84.2 & 9 & 18.8 & 40 & 33.9 & 10 & 2.5 \\
\hline Delusional disorder & F22 & 54 & 10.1 & 33 & 68.8 & 9 & 7.6 & 11 & 2.7 \\
\hline Schizoaffective disorder & F25 & 38 & 7.1 & 0 & 0 & 90 & 76.3 & 20 & 4.9 \\
\hline Bipolar disorder & F30-F31 & 25 & 4.7 & 5 & 10.4 & 24 & 20.3 & 320 & 78.4 \\
\hline
\end{tabular}

Data obtained from NPR for the period 2008-2013 on patients recruited to TOP between 18.10.2002 and 01.09.2014

Abbreviations: ICD-10, International Classification of Diseases, 10th revision, DSM-IV Diagnostic and Statistical Manual of Mental Disorders, 4th edition, SCZ schizophrenia, DEL delusional disorder, SCA schizoaffective disorder, BIP bipolar disorder (type I, type II, and bipolar disorder not otherwise specified) a Percentage of patients within each of the four TOP groups that were registered with each of the four equivalent ICD-10 categories at least once in the NPR during 2008-2013 
Table 3 Agreement statistics between ICD-10 diagnoses in the Norwegian Patient Registry (NPR) and primary diagnoses according to DSM-IV in the Thematically Organized Psychosis (TOP) study

\begin{tabular}{|c|c|c|c|c|c|c|c|c|c|c|c|c|c|}
\hline \multicolumn{2}{|c|}{ TOP-group } & \multicolumn{2}{|c|}{ Agreement $^{\mathrm{a}}$} & \multicolumn{2}{|c|}{ Kappa } & \multicolumn{2}{|c|}{ Sensitivity } & \multicolumn{2}{|c|}{ Specificity } & \multicolumn{2}{|c|}{ PPV } & \multicolumn{2}{|c|}{ NPV } \\
\hline & & Est. & $95 \% \mathrm{Cl}$ & Est. & $95 \% \mathrm{Cl}$ & Est. & $95 \% \mathrm{Cl}$ & Est. & $95 \% \mathrm{Cl}$ & Est. & $95 \% \mathrm{Cl}$ & Est. & $95 \% \mathrm{Cl}$ \\
\hline \multirow[t]{5}{*}{$\overline{S C Z}$} & All & .87 & $.85-.89$ & .74 & $.68-.80$ & .84 & $.81-.87$ & .90 & $.87-.92$ & .88 & $.86-.91$ & .86 & $.83-.89$ \\
\hline & Men & .85 & $.82-.88$ & .69 & $.61-.77$ & .84 & $.81-.88$ & .86 & $.81-.90$ & .89 & $.86-.93$ & .79 & $.74-.84$ \\
\hline & Women & .89 & $.87-.92$ & .77 & $.68-.85$ & .84 & $.78-.89$ & .93 & $.90-.95$ & .87 & $.82-.92$ & .91 & $.88-.9$ \\
\hline & Pre NPR ${ }^{b}$ & .88 & $.85-.91$ & .76 & $.67-.84$ & .85 & $.81-.90$ & .91 & $.87-.94$ & .89 & $.85-.93$ & .87 & $.83-.9$ \\
\hline & Post NPR & .86 & $.83-.89$ & .72 & $.63-.81$ & .83 & $.79-.88$ & .89 & $.85-.93$ & .88 & $.84-.92$ & .85 & $.81-.8$ \\
\hline \multirow[t]{5}{*}{ DEL } & All & .92 & $.90-.93$ & .39 & $.34-.44$ & .69 & $.55-.82$ & .93 & $.92-.95$ & .31 & $.22-.40$ & .99 & $.98-.9$ \\
\hline & Men & .90 & $.88-.92$ & .35 & $.27-.42$ & .61 & $.43-.79$ & .92 & $.89-.94$ & .29 & $.18-.41$ & .98 & $.96-.99$ \\
\hline & Women & .94 & $.92-.96$ & .45 & $.37-.53$ & .82 & $.62-1.0$ & .95 & $.93-.97$ & .33 & $.18-.48$ & .99 & $.99-1$. \\
\hline & Pre NPR ${ }^{b}$ & .94 & $.92-.96$ & .32 & $.24-.39$ & .57 & $.27-.87$ & .95 & $.93-.97$ & .24 & $.09-.40$ & .99 & $.98-1$. \\
\hline & Post NPR ${ }^{c}$ & .90 & $.88-.93$ & .42 & $.34-.49$ & .74 & $.58-.89$ & .91 & $.89-.93$ & .34 & $.23-.45$ & .98 & $.97-.9$ \\
\hline \multirow[t]{5}{*}{ SCA } & Total & .92 & $.91-.94$ & .63 & $.57-.69$ & .76 & $.68-.84$ & .94 & $.93-.96$ & .61 & $.53-.69$ & .97 & $.96-.9$ \\
\hline & Men & .93 & $.91-.95$ & .55 & $.47-.63$ & .69 & $.54-.84$ & .95 & $.93-.97$ & .51 & $.38-.64$ & .98 & $.96-.9$ \\
\hline & women & .92 & $.89-.94$ & .68 & $.60-.77$ & .80 & $.71-.89$ & .93 & $.91-.96$ & .67 & $.57-.77$ & .97 & $.95-.9$ \\
\hline & Pre NPR ${ }^{b}$ & .91 & $.89-.94$ & .59 & $.51-.68$ & .73 & $.61-.85$ & .93 & $.91-.96$ & .57 & $.45-.69$ & .97 & $.95-.9$ \\
\hline & Post NPR ${ }^{c}$ & .93 & $.91-.95$ & .67 & $.59-.75$ & .79 & $.69-.90$ & .95 & $.93-.97$ & .64 & $.53-.75$ & .97 & $.96-.9$ \\
\hline \multirow[t]{5}{*}{ BIP } & Total & .87 & $.85-.89$ & .72 & $.66-.78$ & .78 & $.74-.82$ & .92 & $.90-.94$ & .86 & $.82-.89$ & .88 & $.86-.90$ \\
\hline & Men & .90 & $.87-.92$ & .74 & $.66-.82$ & .78 & $.71-.84$ & .94 & $.92-.96$ & .84 & $.78-.90$ & .92 & $.89-.94$ \\
\hline & women & .85 & $.82-.88$ & .69 & $.60-.77$ & .79 & $.73-.84$ & .90 & $.86-.93$ & .87 & $.82-.91$ & .83 & $.79-.87$ \\
\hline & Pre NPR ${ }^{b}$ & .86 & $.83-.89$ & .68 & $.60-.77$ & .72 & $.66-.78$ & .94 & $.91-.96$ & .88 & $.83-.93$ & .84 & $.81-.88$ \\
\hline & Post NPR ${ }^{c}$ & .89 & $.86-.91$ & .76 & $.67-.84$ & .85 & $.80-.90$ & .91 & $.88-.94$ & .84 & $.78-.89$ & .92 & $.89-.9$ \\
\hline
\end{tabular}

Data obtained from NPR for the period 2008-2013 on patients recruited to TOP between 18.10.2002 and 01.09.2014

Abbreviations: ICD-10 International Classification of Diseases, 10th revision, DSM-IV Diagnostic and Statistical Manual for Mental Disorders, 4th edition, PPV positive predictive value, NPV negative predictive value, Est. estimate, CI confidence interval, SCZ schizophrenia, DEL delusional disorder, SCA schizoaffective disorder, BIP bipolar disorder ${ }^{a}$ Proportion of patients who were either correctly classified as having the diagnosis in question, or correctly classified as not having the diagnosis in question

${ }^{b}$ Patients included in TOP between 18.10 .2002 and 31.12.2007

c Patients included in TOP between 01.01.2008 and 01.09.2014

for the remaining diagnostic groups, kappa was higher among patients recruited in 2008 or later. Schizophrenia had high diagnostic sensitivity irrespective of time of inclusion, while for the remaining three categories the diagnostic sensitivity was higher for patients recruited to TOP concurrently with available NPR-data, i.e. after 01.01.2008, compared to patients recruited in the period 2002-2007. The diagnostic specificity was about $90 \%$ for all diagnostic categories with minimal differences across gender and time of inclusion. For schizophrenia and bipolar disorder, PPV was high across gender and time of recruitment, while for delusional disorder and schizoaffective disorder, PPV was higher among women than men, and higher among patients recruited in 2008 or later compared to patients recruited prior to 2008 .

\section{Diagnostic consistency}

Among the 537 patients in the schizophrenia group, 31,040 out of a total of 36,544 registered contacts with an ICD-10 severe mental disorder diagnosis in the NPR between 2008 and 2013 included a diagnosis of schizophrenia, constituting a diagnostic consistency of $84.9 \%$ (Table 4). Diagnostic consistency was $59.1 \%$ in the delusional disorder group, $65.9 \%$ in the schizoaffective disorder group, and $91.0 \%$ in the bipolar disorder group. In the schizophrenia group, 3.1\% of the severe mental disorder contacts included a diagnosis of persistent delusional disorder, $4.1 \%$ included a diagnosis of schizoaffective disorder, and $1.6 \%$ included a diagnosis of bipolar disorder. In the bipolar disorder group, $1.3 \%$ of the severe mental disorder contacts included a diagnosis of schizophrenia, 1.1\% included a diagnosis of persistent delusional disorder, and $4.1 \%$ included a diagnosis of schizoaffective disorder. Diagnostic consistency for schizophrenia was higher for men (87.5\%) than for women (80.6\%), and diagnostic consistency for delusional disorder was higher for women (72.8\%) than for men (53.0\%) (Table 5). For schizoaffective disorder and bipolar disorder the diagnostic consistency was not different between men and women. The diagnostic consistency was higher in the group of patients included to TOP 
Table 4 Consistency ${ }^{a}$ between ICD-10 diagnoses in the Norwegian Patient Registry (NPR) and DSM-IV diagnosis in the Thematically Organized Psychosis (TOP) study

\begin{tabular}{|c|c|c|c|c|c|c|c|c|c|}
\hline \multicolumn{2}{|l|}{ TOP group } & \multicolumn{2}{|c|}{$\mathrm{SCZ}(n=537)$} & \multicolumn{2}{|c|}{$\operatorname{DEL}(n=48)$} & \multicolumn{2}{|c|}{ SCA $(n=118)$} & \multicolumn{2}{|c|}{$\mathrm{BIP}(n=408)$} \\
\hline NPR diagnoses & ICD-10 codes & $n$ & $\%$ & $n$ & $\%$ & $n$ & $\%$ & $n$ & $\%$ \\
\hline Severe mental disorder & F20-F31, F32.3, F33.3 & 36,544 & 100 & 1661 & 100 & 6322 & 100 & 13,238 & 100 \\
\hline Schizophrenia & F20 & 31,040 & 84.9 & 523 & 31.5 & 1040 & 16.5 & 174 & 1.3 \\
\hline Delusional disorder & F22 & 1123 & 3.1 & 982 & 59.1 & 186 & 2.9 & 146 & 1.1 \\
\hline Schizoaffective disorder & F25 & 1509 & 4.1 & 0 & 0 & 4168 & 65.9 & 543 & 4.1 \\
\hline Bipolar disorder & F30-F31 & 599 & 1.6 & 66 & 4.0 & 695 & 11.0 & 12,044 & 91.0 \\
\hline
\end{tabular}

Abbreviations: ICD-10 International Classification of Diseases, 10th revision, DSM-IV Diagnostic and Statistical Manual of Mental Disorders, 4th edition, TOP Thematically Organized Psychosis, SCZ schizophrenia, DEL delusional disorder, SCA schizoaffective disorder, BIP bipolar disorder (type I, type II, and bipolar disorder not otherwise specified)

a Percentage of all registered contacts with a severe mental disorder diagnosis (F20-F31, F32.3, F33.3) in the NPR during 2008-2013 that included the primary DSM-IV diagnosis assigned when recruited to the TOP-study between 18.10 .2002 and 01.09.2014

prior to than after January 1st 2008 for all diagnostic groups (Table 5).

\section{Discussion}

Among 1111 men and women with a diagnosis of schizophrenia, delusional disorder, schizoaffective disorder, or bipolar disorder, we found high degree of agreement between DSM-IV diagnoses as determined by structured diagnostic interview in a clinical research setting and ICD-10 diagnoses as determined by the treating clinicians and registered in the national patient registry. For schizophrenia and bipolar disorder, eight out of ten patients received the equivalent ICD-10 and DSM-IV diagnoses, with minimal differences between men and women. For bipolar disorder, diagnostic agreement was better for patients recruited in 2008 or later, while for schizophrenia the agreement was similar across time. For delusional and schizoaffective disorder patients the proportion of correctly diagnosed patients in the NPR ranged between 57 and 80\%, with higher proportion for women compared to men, and higher proportion for patients recruited to the TOP study during the time NPRdata was available at individual level (2008-2013) compared to the period for which NPR data was not available (2002-2007). Poorer agreement among patients recruited prior to 2008 may be explained by some patients being lost to follow-up because they did not need treatment in specialist health care after inclusion.

The results indicate that diagnostic data from NPR show good agreement for severe mental disorders in general, but the level of agreement was best for schizophrenia (kappa 0.74) and bipolar disorder (kappa 0.72), fair for schizoaffective disorder (kappa 0.63), and poor for delusional disorder (kappa 0.39). Validity studies of registry data in other Nordic countries have also shown good agreement for schizophrenia [2-7, 17], but somewhat poorer agreement for schizoaffective disorder [2]. The poorer agreement for delusional and schizoaffective disorder in the current study may be explained by the low prevalence of the disorders in the TOP sample. Kappa reliability is dependent on prevalence of the disorder in addition to sensitivity and specificity [18]. Poorer agreement for schizoaffective disorder than for schizophrenia may also be explained by inherent differences in diagnostic criteria between ICD-10 and DSM-IV.

Few studies have investigated the validity of bipolar disorder diagnoses. In a previous study from Northern Norway, Øiesvold and colleagues demonstrated that diagnostic concordance for bipolar disorders was moderate (kappa ranging from 0.41 to 0.47 ) based on expert

Table 5 Consistency (\%) between ICD-10 diagnoses in the Norwegian Patient Registry (NPR) and DSM-IV diagnosis in the Thematically Organized Psychosis (TOP) study across gender and time

\begin{tabular}{|c|c|c|c|c|}
\hline & SCZ & DEL & SCA & $\mathrm{BIP}$ \\
\hline All patients $(n=1111)$ & 84.9 & 59.1 & 65.9 & 91.0 \\
\hline Men $(n=577)$ & 87.5 & 53.0 & 65.1 & 92.6 \\
\hline Women $(n=534)$ & 80.6 & 72.8 & 66.4 & 90.0 \\
\hline Patients included in TOP between 18.10.2002 and 31.12.2007 $(n=530)$ & 88.5 & 87.1 & 75.9 & 93.5 \\
\hline Patients included in TOP between 01.01.2008 and 01.09.2014 $(n=581)$ & 81.2 & 52.3 & 56.2 & 89.4 \\
\hline
\end{tabular}

Data obtained from NPR for the period 2008-2013 on patients recruited to TOP between 18.10.2002 and 01.09.2014

Abbreviations: ICD-10 International Classification of Diseases, 10th revision, DSM-IV Diagnostic and Statistical Manual of Mental Disorders, 4th edition, SCZ schizophrenia, DEL delusional disorder, SCA schizoaffective disorder, BIP bipolar disorder (type I, type II, and bipolar disorder not otherwise specified)

a Percentage of all registered contacts with a severe mental disorder diagnosis (F20-F31, F32.3, F33.3) in the NPR that included the primary DSM-IV diagnosis assigned when recruited to the TOP-study 
diagnostic interviews of a sample of 250 first-time admitted patients at a regional hospital [19]. Their study demonstrated higher concordance for manic episode than for bipolar depression, which indicates higher validity for bipolar disorder type I than bipolar disorder type II diagnoses in the NPR. In comparison, we found close to $80 \%$ agreement (kappa 0.72 ) for the total bipolar disorder group.

Patients diagnosed with schizophrenia or bipolar disorder based on a structured interview and review of case notes received the corresponding diagnosis at 85 and $91 \%$, respectively, of registered contacts for severe mental disorders. Moreover, there were minimal overlap between schizophrenia and bipolar disorder, i.e. for schizophrenia only a few percent of the registered contacts included a diagnosis of bipolar disorder and vice versa. Hence the findings indicate that clinicians are consistent in their differentiation between schizophrenia spectrum disorders and bipolar disorders.

\section{Strengths and limitations}

The major strength of this study lies in the high number of rigorously assessed patients recruited from several hospitals during a period of 12 years. Since the TOPstudy was initiated several years before subject-specific data were available from the NPR the linked dataset allowed for study of time-sensitive agreement rates. However, the results must be interpreted with the following limitations in mind. Firstly, all patients in the TOP-study were recruited from hospitals and outpatient clinics, and the TOP interviewers were not blinded for clinical diagnoses. After inclusion the referring clinician received a written report which included diagnostic information. Thus, for most patients the two diagnostic assessments were not independent, which may have inflated the diagnostic agreement and consistency estimates. However, by investigating differences in diagnostic agreement and consistency across time, we were able to evaluate the putative time-dependent effect of this dependency. For the major diagnostic categories schizophrenia and bipolar disorder, there were only minor differences in agreement across time. Secondly, while the diagnoses in TOP are given according to DSM-IV, diagnoses in NPR are given according to ICD10. Due to structural differences in the delineation of bipolar disorder between DSM-IV and ICD-10, we were not able to distinguish between bipolar disorder type I and type II in the present study. However, our findings indicate a high degree of accuracy for bipolar disorder diagnoses in general in the NPR. Thirdly, since only patients recruited to the TOP study were included in the present study, the results are not necessarily representative for all patients diagnosed with severe mental disorders in the NPR.

\section{Conclusions}

More than eight out of ten patients diagnosed with schizophrenia or bipolar disorder in a clinical research project received the same diagnosis by their treating clinician as registered in the NPR. Moreover, the diagnostic consistency, i.e. proportion of severe mental disorder registrations in NPR with the equivalent ICD-10 diagnosis as the primary DSM-IV diagnosis given in the TOP-study, was $85 \%$ for schizophrenia and $91 \%$ for bipolar disorder. There were minimal differences in diagnostic agreement and consistency between genders and across time, and negligible diagnostic overlap between affective and nonaffective disorders. The results support the use of registrybased diagnoses of schizophrenia and bipolar disorder when searching for cases in epidemiological and genetic studies involving severe mental disorders.

\section{Abbreviations}

DSM-IV: Diagnostic and Statistical Manual of Mental Disorders, 4th version; ICD-10: International Classification of Diseases, 10th revision; NPR: Norwegian Patient Registry; TOP-study: Thematically Organized Psychosis study

\section{Acknowledgements}

We thank the administrative staff at the NPR for support in retrieval of data for this study.

\section{Funding}

This study was funded by the Norwegian Institute of Public Health, the Research Council of Norway (223273) and KG Jebsen Foundation. The funding bodies provided financial support for salary, data collection, and research infrastructure as described in the study protocol submitted with the funding proposal. The funding bodies took no part in analysis or interpretation of data.

\section{Availability of data and materials}

The datasets generated during and/or analyzed during the current study are not publicly available due to data protection regulations.

\section{Authors' contributions}

RN planned the study, analyzed the data, interpreted the results, and wrote the first draft of the manuscript. EGJ planned the study, interpreted the results, and contributed to writing the manuscript. IJB analyzed the data, interpreted the results, and contributed to writing the manuscript. GPK planned the study and interpreted the data. TRK planned the study, interpreted the data, and contributed to writing the manuscript. IM planned the study, interpreted the data, and contributed to writing the manuscript. OAA planned the study, interpreted the data, and contributed to writing the manuscript. All authors read and approved the final manuscript.

\section{Competing interests}

The authors declare that they have no competing interests.

\section{Consent of publication}

Not applicable

\section{Ethics approval and consent to participate}

The study was approved by the Regional Committee for Medical and Health Research Ethics South Eastern Norway, P.O. Box 1130, Blindern, N-0318 Oslo, Norway, reference number \#2009/2485, extension date Dec 20, 2013, and was performed in accordance with the ethical standards laid down in the 1964 Declaration of Helsinki and its later amendments.

Informed consent was obtained from all participants before they were included in the study. All participants were assessed by psychiatrists, psychologists or medical doctors in psychiatric training, that were specifically trained in the study's assessment protocol. In the evaluation of the participants' competence to give informed consent, a particular emphasis 
was made on the degree of disorganization and lack of insight. The participants were also given a copy of the assessment form to take home before signing, to study it without disturbance and/or ask opinion of others if they wanted. Before signing, the member of the assessment team went through the consent form with the participant, particularly ensuring that he or she was fully aware they were participating in a research study, what their consent meant in regards of sharing data and their right to withdraw from the study at any time without consequences. No participants were included in the study based on consent from family members alone. These criteria for obtaining informed consent were approved by the Regional Committee for Medical and Health Research Ethics South Eastern Norway, ref \#2009/2485, extension date Dec 20, 2013

\section{Publisher's Note}

Springer Nature remains neutral with regard to jurisdictional claims in published maps and institutional affiliations.

\section{Author details}

${ }^{1}$ Norwegian Institute of Public Health, P.O. Box 4044Nydalen, N-0403 Oslo, Norway. ${ }^{2}$ Norwegian Center for Mental Health Research (NORMENT), Oslo University Hospital \& University of Oslo, P.O. Box 4956Nydalen, N-0424 Oslo, Norway. ${ }^{3}$ Department of Clinical Neuroscience, Centre of Psychiatric Research, Karolinska Institutet, Administration, Tomtebodavägen 18A, 5th floor, 17177 Stockholm, Sweden. ${ }^{4}$ Institute of Clinical Medicine, University of Oslo, P.O. Box 1171Blindern, N-0319 Oslo, Norway.

Received: 10 September 2016 Accepted: 8 March 2017 Published online: 14 March 2017

\section{References}

1. Ludvigsson JF, Andersson E, Ekbom A, Feychting M, Kim JL, Reuterwall C, Heurgren M, Olausson PO. External review and validation of the Swedish national inpatient register. BMC Public Health. 2011:11:450.

2. Jakobsen KD, Frederiksen JN, Hansen T, Jansson LB, Parnas J, Werge T. Reliability of clinical ICD-10 schizophrenia diagnoses. Nord J Psychiatry. 2005;59:209-12.

3. Dalman C, Broms J, Cullberg J, Allebeck P. Young cases of schizophrenia identified in a national inpatient register-are the diagnoses valid? Soc Psychiatry Psychiatr Epidemiol. 2002;37:527-31.

4. Ekholm B, Ekholm A, Adolfsson R, Vares M, Osby U, Sedvall GC, Jönsson EG. Evaluation of diagnostic procedures in Swedish patients with schizophrenia and related psychoses. Nord J Psychiatry. 2005;59:457-64.

5. Pihlajamaa J, Suvisaari J, Henriksson M, Heilä H, Karjalainen E, Koskela J, Cannon M, Lönnqvist J. The validity of schizophrenia diagnosis in the Finnish Hospital Discharge Register: findings from a 10-year birth cohort sample. Nord J Psychiatry. 2008;62:198-203.

6. Isohanni M, Mäkikyrö T, Moring J, Räsänen P, Hakko H, Partanen U, et al. A comparison of clinical and research DSM-III-R diagnoses of schizophrenia in a Finnish national birth cohort. Clinical and research diagnoses of schizophrenia. Soc Psychiatry Psychiatr Epidemiol. 1997:32:303-8.

7. Mäkikyrö T, Isohanni M, Moring J, Hakko H, Hovatta I, Lönnqvist J. Accuracy of register-based schizophrenia diagnoses in a genetic study. Eur Psychiatry. 1998;13:57-62.

8. Weiser M, Werbeloff N, Dohrenwend BP, Levav I, Yoffe R, Davidson M. Do psychiatric registries include all persons with schizophrenia in the general population? A population-based longitudinal study. Schizophr Res. 2012;135:187-91.

9. World Health Organization. The ICD-10 Classification of Mental and Behavioural Disorders. Clinical Descriptions and Diagnostic Guidelines. Geneva: World Health Organization; 1992.

10. Surén P, Bakken IJ, Aase H, Chin R, Gunnes N, Lie KK, et al. Autism spectrum disorder, ADHD, epilepsy, and cerebral palsy in Norwegian children. Pediatrics. 2012;130:e152-8.

11. Nesvåg R, Knudsen GP, Bakken IJ, Høye A, Ystrøm E, Surén P, et al. Substance use disorders in schizophrenia, bipolar disorder, and depressive illness: a registry-based study. Soc Psychiatry Psychiatr Epidemiol. 2015;50:1267-76.

12. Fadum EA, Stanley B, Qin P, Diep LM, Mehlum L. Self-poisoning with medications in adolescents: a national register study of hospital admissions and readmissions. Gen Hosp Psychiatry. 2014;36:709-15.

13. Engh JA, Friis $S$, Birkenaes $A B$, Jónsdóttir $H$, Klungsøyr $O$, Ringen $P A$, et al. Delusions are associated with poor cognitive insight in schizophrenia. Schizophr Bull. 2010;36:830-5.
14. American Psychiatric Association. Diagnostic and Statistical Manual of Mental Disorders. fourthth ed. Washington, D.C.: American Psychiatric Association Press; 1994.

15. First MB, Spitzer RL, Gibbon M, Williams JBW. Structured Clinical Interview for DSM-IV-TR Axis I Disorders, Research Version, Patient Edition. (SCID-I/P, Version 2.0). New York: Biometrics Research, New York State Psychiatric Institute; 1995

16. Cohen J. A coefficient of agreement for nominal scales. Educ Psychol Meas. 1960:20:37-46

17. Vares M, Ekholm A, Sedvall GC, Hall H, Jönsson EG. Characterization of patients with schizophrenia and related psychoses: evaluation of different diagnostic procedures. Psychopathology. 2006;39:286-95.

18. Faraone SV, Tsuang MT. Measuring diagnostic accuracy in the absence of a "Gold standard". Am J Psychiatry. 1994;151:650-7.

19. Øiesvold T, Nivison M, Hansen V, Sørgaard KW, Østensen L, Skre I. Classification of bipolar disorder in psychiatric hospital. a prospective cohort study. BMC Psychiatry. 2012;12:13.

\section{Submit your next manuscript to BioMed Central and we will help you at every step:}

- We accept pre-submission inquiries

- Our selector tool helps you to find the most relevant journal

- We provide round the clock customer support

- Convenient online submission

- Thorough peer review

- Inclusion in PubMed and all major indexing services

- Maximum visibility for your research

Submit your manuscript at www.biomedcentral.com/submit
) Biomed Central 